\title{
Analysis of Programming Language and Software Development by Computer \\ Xu Pei
}

Nanchang Institute of Science \& Technology, Nanchang, 330108

Keywords: Computer language; Software development; Object-oriented; Process-oriented

\begin{abstract}
When human society entered into the information age, with the rapid development of science and technology, computer technology has been widely applied in various industries, and that has also promoted the development of computer application software. The development of computer software is a complex work. The relevant enterprises should choose the most appropriate programming language according to the application software they used to improve the efficiency of software development, ensure the quality of software and improve their overall competitiveness.
\end{abstract}

\section{Introduction}

Entering the 21st century, software has already become a part of our work, study and life, software industry has also become a young industry full of vigour, vitality and creative spirit which is very popular with youngsters and has got the focus of the society. This has promoted more people to join in software development industry. Based on analysis of development of software industry in our country, compared with the abroad, the software industry of our country still has a long way to go. It is the main problem to lack of the applied and researching type talents in software industry of our country, which led many people to work in this industry for a time not very long, they would move up to other industries after working for several years, even for a period of time in this industry. Therefore, many technologies can not be effectively summarized and used, and even be forgotten. This paper makes a detailed analysis to the reason and expresses the effect of programming language on the software industry by investigating and researching the popular programming language in modern society.

\section{Programming Language}

Programming language is refer to the formal language used to define the computer program. It is a standardized communication kill used to issue instructions to computer. A computer language can help programmer to precisely define the data used by a computer and precisely define the actions taken under different conditions.

The earliest programming language was generated after the invention of computer. At that time, it was used to control the actions of a jacquard loom and a player piano. Now, hundreds of different programming languages have been invented throughout the computing world, and there are still new programming languages to be invented each year. Many programming languages need to explain the calculation program through the instruction mode. But some programming languages are declarative programming, and which only explain the results required rather than the calculation program.

Programming language is commonly known as "computer language". There are many kinds of programming languages. Generally, it can be divided into three major classes machine languages, assembly languages and high-level languages. All actions and steps of computer are executed in accordance with the program programmed with computer language. Program is a set of instructions executed by a computer, and which is programmed with the language we mastered. Therefore, we can control a computer by giving instructions to it with computer language. The development sequence of computer language is low-level language, specific language, assembly language and high-level language, as shown in Fig.1 . 


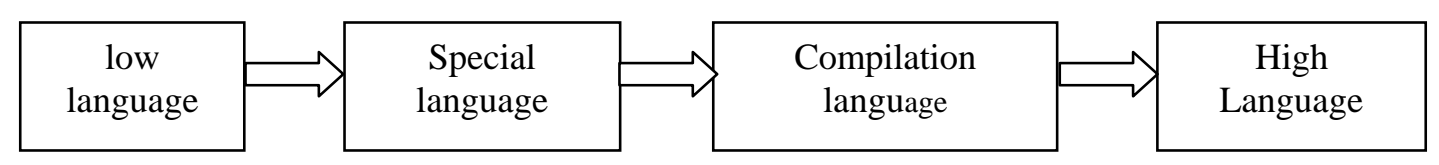

Figure 1. Development of Programming Language

\section{Common Computer Language}

Assembly Languages. In order to ease the pain due to the using of machining language programming, a beneficial improvement is made by people that some simple English alphabets, symbol strings are used to replace the binary strings of a specific instruction. For example, "ADD" is used to represent addition, "MOV" is used to represent the data transmission. By dong this, people easily read and understand the actions of program, and error correction and maintenance also become more convenient. This programming language is known as assembly language, i.e., the second generation of computer language.

Although with high efficiency, assembly language still relies heavily on the machine hardware due to the bad portability. The function and speciality of computer hardware can be exactly developed with the assembly language programmed aiming at the specific hardware of computer, and the program has simple structure and high quality. Therefore, the assembly language program is a frequent and powerful tool for software development.

High-Level Language. High-level languages includes BASIC (True basic, Qbasic, Virtual Basic), $\mathrm{C}$, computer language $\mathrm{C}++$, PASCAL, FORTRAN, intelligent languages (LISP, Prolog, CLIPS, OpenCyc, Fazzy), dynamic languages (Python, PHP, Ruby, Lua) etc. The source program of high-level languages can be executed with the means of explanation and translate \& edit. Generally, the latter is used for execution.

High-level language is a choice for most programmers. Compared with assembly language, it can not only synthesize many relevant machine instructions into the single instructions but also remove the details related to the specific operation rather than completion work. For example, stacks and register are used to greatly simplify the instructions of program. For the reason of omitting many details, too much professional knowledge is not required for programmers. High-level language is a collective name of many programming languages relative to assembly language, it is not a specific language. It includes many programming languages such as the popular VB, VC, FoxPro and Delphi. These languages have different syntax and command formats.

\section{High-Level Overview for Computer Software Development}

Object-Oriented. The object oriented program design and data abstraction are really important in modern program designing idea. The future languages programmed will be the full objects oriented rather than the simple languages which are more easy to express the real world and be programmed by people. In addition to the professional programmers, people also can use computer language for programming in a simple way as customizing a work process in the real life.

For object oriented, the simple mechanisms and dynamic interface models are provided. Modularization and information hiding are realized for the encapsulating state variable and the corresponding method; providing the prototype of the first-class objects, the code reuse is achieved through an inheritance mechanism that the subclass can use the method provided by the super class.

Process-Oriented. It is the programming idea of process oriented to use modules as the central and apply the designing software for process oriented. The program shall be divided into several modules by function which are used to form the tree structures, and these tree structures represent the calling relations between different modules; the relationship between different modules shall be as simple as possible, and has independent function; every module consists of some basic structures such as sequence, selection and loop. It is the specific method for implementation to use the subprogram.

Process oriented is developed from top to bottom, and is a process of stepwise refinement. Firstly, a complex big system shall be divided into many independent small modules, and the mechanism 
for assembly and interaction between every module shall be defined separately. After the completion of design and development of these submodules, the overall assembly shall be completed for completion of the big system.

\section{Effect of Different Computer Languages on Development of Computer Application Software}

Among these computer programming languages, $\mathrm{C}$ language has the best portability and broad scope of application which can be operated in most programs, and it is appropriate difficulty. Therefore, programmers frequently use this programming language in software development process. For the shortcomings of $\mathrm{C}$ language, programmers can choose other programming languages for hybrid programming to overcome the shortcomings of $\mathrm{C}$ language. By this way, the efficiency and quality of software development shall be ensured. Compared with $C$ language, the learning difficulty of Visual Basic language is relatively low, but it has some shortcomings, for example, its efficiency in executing program is very low.

In the process of software development, assembly language is relatively suitable for the primary programming. Because the learning difficulty of assembly language is relatively large, and the language structure is very complex when it is used for programming. But assembly language has higher execution efficiency.

Programming languages are the tool for computer software development. Developers can choose one or more programming languages for developing software from many programming languages. The selection of programming languages may directly affect the programming efficiency and the service efficiency of the computer software. So it is very important for improvement of software development efficiency to choose a appropriate programming language for programming. In this way, the software developed will be more simple and convenient.

The language shall be chose according to the situations of the actual software to be developed. Sometimes, the single programming language can not help us to finish our work for software development. Therefore, it is very necessary for us to deeply understand the programming languages in different fields. In the process of software development, it is very important for the improvement of work efficiency and the shortening of development time of software to be familiar with the programming languages, and the corresponding programming shall be realized according to the application area of software. Software is mainly used for the repeatable manual part, so the programming language shall be chose conveniently and flexibility.

\section{Conclusion}

With the rapid progress and development of computer, programming language and software development also are continuously developed. The languages are all products of our time from the machine language ago to the high-level language now. For example, $\mathrm{C}++$ and JAVA are developed from $\mathrm{C}$ language ago. The development of programming language and software development will help to promote the development of computer industry and improvement of scientific and technological strength. So it is required for the research specialist staff to constantly understand the advantages \& disadvantages and the scope of use of every programming language. Only in this way, programmers can develop the excellent computer application software and realize the best work results by reasonably using the programming language.

\section{References}

[1] Ribeiro A, Silva A R D. Evaluation of XIS-Mobile, a Domain Specific Language for Mobile Application Development[J]. Journal of Software Engineering \& Applications, 2014, 7(11):906-919.

[2] Lou D. Computer-Based Software Development JAVA Programming Language Inquiry[J]. Wireless Internet Technology, 2015. 
[3] Winetzhammer S, Westfechtel B. Compiling graph transformation rules into a procedural language for behavioral modeling[C]// International Conference on Model-Driven Engineering and Software Development. IEEE, 2015:415-424.

[4] $\mathrm{Hu} \mathrm{X}$. Application Value of JAVA Programming Language in Computer Software Development[J]. China Computer \& Communication, 2017.

[5] Ivanova V, Sedov B, Sheynin Y, et al. Domain-specific languages for embedded systems portable software development[C]// Open Innovations Association. IEEE, 2014:24-30.

[6] Badreddin O, Forward A, Lethbridge T C. A test-driven approach for developing software languages[C]// International Conference on Model-Driven Engineering and Software Development. IEEE, 2014:225-234.

[7] Ivanova, V, Sedov, B, Sheynin, Y, et al. Domain-specific languages for embedded systems portable software development[J]. 2014:24-30.

[8] Lavazza L, Morasca S, Tosi D. An empirical study on the effect of programming languages on productivity[C]// ACM Symposium on Applied Computing. ACM, 2016:1434-1439.

[9] Zhang $X$, University $H$ N. The Influence of Different Programming Languages on the Development of Computer Software[J]. Computer \& Telecommunication, 2016.

[10]Lavazza L, Morasca S, Tosi D. An empirical study on the effect of programming languages on productivity[J]. 2016:1434-1439.

[11]Garrido J M. Improving Software Development for Embedded Systems[C]// Southeast Conference. ACM, 2017:231-234.

[12]Long M, Amp H R. An Analysis of the Algorithm for "Spending 100 Dollars on 100 Chickens" in C Programming Language[J]. Software Engineering, 2017. 